\title{
Type-specific human papillomavirus infections and Pap test findings in Inuit and non-Inuit women in Nunavut, Canada
}

\author{
Totten $\mathrm{S}^{1^{*}}$, Severini $\mathrm{A}^{2}$, Jayaraman $\mathrm{GC}^{3}$, Faybush $\mathrm{ST}^{2}$, Johnson $\mathrm{G}^{4}$, Demers $A A^{1}$, Sobol $\mathrm{I}^{5}$, Mao $\mathrm{Y}^{3}$, Wong $\mathrm{T}^{1}$ \\ ${ }^{1}$ Centre for Communicable Diseases and Infection Control, Public Health Agency of Canada, Ottawa, ON \\ ${ }^{2}$ National Microbiology Laboratory, Public Health Agency of Canada, Winnipeg, MB \\ ${ }^{3}$ Centre for Chronic Disease Prevention, Public Health Agency of Canada, Ottawa, ON \\ ${ }^{4}$ DynaLIFE $E_{D x}$, Edmonton, $A B$ \\ ${ }^{5}$ Nunavut Department of Health and Social Services, Nunavut
}

${ }^{*}$ Correspondence: stephanie.totten@phac-aspc.gc.ca

\begin{abstract}
Objective: To determine the prevalence and distribution of type-specific human papillomavirus (HPV) infections and their association with cytological outcomes in women living in the Canadian territory of Nunavut.

Methods: Surveillance of type-specific HPV infection was conducted. Cervical specimens of all Inuit, First Nations and non-Aboriginal women in Nunavut who presented for a Pap test in any clinical setting between January 2008 and March 2009 were tested for HPV infection. The association between high-grade cervical lesions and HPV type was also examined.
\end{abstract}

Results: HPV results were available for 4,043 individual women (13 to 77 years). Of those with known ethnicity $(\mathrm{N}=4,033), 89.2 \%$ were Inuit, $0.4 \%$ were First Nations and $10.4 \%$ were non-Aboriginal. First Nations women were included in all analyses except those making comparisons by ethnicity, due to the small number of individuals in this group. Overall, $29.9 \%$ of women were found to be infected with HPV (any type) and $19.9 \%$ with any high-risk HPV (type 16, 18, 31, 33, 35, 39, 45, 51, 52, 56, 58 or 59). Most often, women were infected with HPV 16 (6.4\%) followed by HPV 31 (3.1\%). There were no statistically significant differences between Inuit and non-Aboriginal (reference group) women 20 years of age and older regarding the prevalence of any HPV (odds ratios (OR): $1.19,95 \%$ confidence intervals $(\mathrm{Cl}): 0.92-1.54)$, high-risk HPV (OR: $1.06,95 \% \mathrm{Cl}$ : 0.78-1.44) or HPV 16 and 18 (OR: $0.81,95 \% \mathrm{Cl}: 0.51-1.27)$. HPV 31 was the only type that was significantly more frequent among Inuit than non-Aboriginal women (OR: $3.95,95 \% \mathrm{Cl}$ : 1.24-12.54). There was no difference in the overall occurrence of cervical abnormalities between non-Aboriginal and Inuit women ( $p$-value $=0.17$ ). HPV 16 was strongly associated with cervical dysplasia, being present in $50.9 \%$ of specimens with a high-grade lesion.

Conclusion: HPV is a significant public health issue in the territory of Nunavut. The findings presented in this article are similar to those in other studies among Inuit women, with prevalence of HPV being higher than in studies conducted among non-Inuit women in other regions of Canada. These results provide a baseline of HPV prevalence that precedes the introduction of the Nunavut HPV Immunization Program in 2010 and will allow for future evaluation. The high prevalence of HPV infection among women living in Nunavut can be reduced through immunization and associated high-grade cervical abnormalities mitigated by regular cervical screening.

\section{Introduction}

Human papillomavirus (HPV), a necessary cause of cervical cancer, is estimated to be one of the most common sexually transmitted infections in Canada $(1,2)$. Two effective preventive vaccines have been developed and are available in Canada. The quadrivalent vaccine that protects against non-oncogenic (low-risk) HPV 6 and 11 and oncogenic (high-risk) HPV 16 and 18 was approved for use in Canada in 2006 and is offered to girls in all provinces and territories in Canada as a part of publicly-funded school-based immunization programs. In 2010 a bivalent vaccine against HPV 16 and 18 was approved for use, but it is not currently offered through publiclyfunded immunization programs. 
The territory of Nunavut has a young, mainly Inuit population of approximately 30,000 that is sparsely distributed across two million square kilometers in 25 communities in the circumpolar region of Canada. There are challenges in health care and public health in Nunavut. For example, compared to Canadian averages, its inhabitants are disproportionately affected by poor birth outcomes, lower life expectancy and chronic and infectious diseases, including high rates of sexually transmitted infections (3). For example, reported rates of chlamydia in Nunavut are 15 times higher than the national average and gonococcal infections are 50 times higher (4). Elevated cervical cancer incidence rates have been reported in Inuit women compared to non-Inuit women in Nunavut (8.0 vs. 5.7 per 100,000 population) (5). Development of public health strategies and programs in Nunavut aims to improve health outcomes (6), including the implementation of publicly-funded school-based HPV immunization in March 2010 for girls in grade 6 (11 to 12 years of age).

There is a paucity of data on the prevalence and type distribution of HPV infections among women in Nunavut. In 1999, a cross-sectional study that included 1,290 women in 19 Nunavut communities reported a prevalence of high-risk HPV of $26 \%$ and a prevalence of squamous intraepithelial lesions of $7.2 \%(7,8)$. These authors did not report their results by HPV type. Studies in other regions of Canada have found overall HPV prevalence rates that vary depending on population: $10.8 \%$ in women aged 18-69 in Newfoundland (9); $16.8 \%$ among women aged $13-$ 86 in British Columbia (10); $21.8 \%$ among female university students in Montreal (11); $13.3 \%$ among women aged $15-49$ in Ontario (12); 28.9\% among Inuit women aged 15-69 in Nunavik, Quebec (13); 33\% in women in Winnipeg (age range not reported) (14); and 24.2\% among women aged 14-85 in the Northwest Territories (15).

In response to a need for baseline epidemiological data to evaluate the effectiveness of implementing a publiclyfunded HPV immunization program for females, Nunavut public health authorities conducted surveillance from January 2008 to March 2009 with the objectives of determining the prevalence and distribution of HPV types in Inuit and other women living in Nunavut and examining the association between type-specific infection and cervical cytology results. This report provides the results from this surveillance initiative and the above objectives. All geographic regions of Nunavut were included in order to inform clinicians and public health practitioners throughout the territory.

\section{Methods}

Public health HPV surveillance was initiated in January 2008 under the authority of the Public Health Act and Regulations as executed by Nunavut's Chief Medical Officer of Health. All females presenting to any community, public health, or hospital-based clinic for cervical screening in Nunavut between January 2008 and March 2009 were included. In Nunavut, ethnicity is self-identified when individuals register with the territorial universal health care insurance plan (e.g., at birth or immigration).

Liquid-based cervical specimens were collected using BC Surepath collection kits and processed as per standard practice at the Cytopathology Laboratory at DynaLIFEDx. Leftover aliquots were forwarded to the National Microbiology Laboratory (NML) of the Public Health Agency of Canada for HPV typing. An in-house Luminex test that detects 45 HPV types was used for the HPV typing, with amplification by nested polymerase chain reaction (PGMY) primers and GP5+/GP6+) (16). The NML-Luminex method compares favourably to the commercially available Roche LinearArray genotyping method, but fewer types are detected in samples with multiple infections with more than three types. However, in this population only $3 \%$ of positive specimens (about $1 \%$ of the participants) were positive for more than three HPV types, and therefore any difference in type distribution from the Roche LinearArray would be negligible. The rare type HPV 97 was not detected by the version of the NMLLuminex method used for this study (16). HPV types were analysed according to species (as per deVilliers et al) and grouped by carcinogenic potential as assessed by the International Agency for Research on Cancer Monograph Working Group (high-risk: HPV 16, 18, 31, 33, 35, 39, 45, 51, 52, 56, 58, 59; and probably/possibly carcinogenic: HPV 68, 26, 53, 66, 67, 70, 73, 82, 30, 34, 69, 85, 97) $(17,18)$. HPV types were also analysed by species, in order to identify infections that include oncogenic types genetically related to the vaccine types HPV 16 and HPV 18 (species a09 and a07, respectively). Within each species, HPV types show similar biological properties and there is some evidence of cross-protection following vaccination (19).

Cytological findings were categorized using the Bethesda System: negative for intraepithelial lesion or malignancy; atypical squamous cells of undetermined significance (ASCUS); atypical squamous cells - cannot 
exclude high-grade squamous epithelial lesion (ASC-H); low-grade squamous intraepithelial lesion (LSIL); highgrade squamous epithelial lesion (HSIL); squamous cell carcinoma (SCC); atypical glandular cells, not otherwise specified (AGC) (20). Cytological and HPV results were linked by a unique identifier assigned to each specimen and a sub-identifier unique to each individual was used to identify women who had more than one Pap test during the surveillance period. When more than one specimen was available for a participant, only one randomly-picked specimen was included in order to estimate the prevalence among the individual women tested, rather than among the number of specimens. Comparisons between Inuit and non-Aboriginal women were made using an age-adjusted logistic regression model with non-Aboriginal as the reference group. Data on First Nations women were not part of this analysis.

The association between the Pap result and HPV type was calculated using odds ratios (OR) and 95\% confidence intervals $(95 \% \mathrm{Cl})$. The outcomes of particular interest were cytological results most likely to lead to invasive cervical cancer. To this end, ASC-H, AGC, HSIL and SCC results were combined to create a category of highgrade cervical lesions. All other cervical cytology findings were combined as low-grade cervical lesions in the logistic regression model. Specimens without valid cytology results were excluded from this analysis, but were still tested for HPV.

\section{Results}

A database of 4,683 records with valid participant identification number, cytology and HPV results was created, representing 4,043 individual females (640 "repeat" testers). Of those with known ethnicity $(n=4,033), 3,596$ were identified as Inuit (89.2\%), 17 as First Nations (0.4\%) and 420 as non-Aboriginal (10.4\%).

Of those with known age ( $n=3,877)$, the median was 30 years (range: 13 to 77$), 32.8 \%$ were under the age of 25 . Inuit women were younger than non-Aboriginal women (median age: 29 vs. 35 years, respectively; $p<0.001$ ).

\section{Prevalence of HPV and cervical cytological abnormalities}

Of the 4,043 unique individuals, 1,207 (29.9\%) tested positive for at least one HPV type, 19.9\% were positive for at least one high-risk HPV and $7.5 \%$ were positive specifically for types 16 or 18 , which are included in the commonly used vaccines (Table 1). In addition, $6.4 \%$ were positive for at least one probably carcinogenic HPV type and $9.5 \%$ tested positive for multiple HPV infections. Women under 25 years of age had the highest prevalence of HPV with $45.4 \%$ being HPV positive, compared to $30.9 \%$ in those aged 25 to $29,23.3 \%$ in those aged 30 to 39 and $16.2 \%$ in those aged 40 and older.

Table 1: Prevalence of human papillomavirus (HPV) infection by age group among women presenting for cervical cancer screening in Nunavut, January 2008 to March 2009 (n=4,043)

\begin{tabular}{|c|c|c|c|c|c|c|}
\hline \multicolumn{2}{|r|}{ Age: $\mathbf{N}$} & \multicolumn{5}{|c|}{ HPV Infection: N (\%) } \\
\hline $\begin{array}{l}\text { Age } \\
\text { Group }\end{array}$ & N for Age Group & Any type & Multiple & High-risk $^{1}$ & $\begin{array}{c}\text { Probablyl } \\
\text { possibly } \\
\text { carcinogenic }^{2}\end{array}$ & HPV 16/18 \\
\hline$<20$ & 521 & $244(46.8)$ & $101(19.4)$ & $183(35.1)$ & $58(11.1)$ & $81(15.5)$ \\
\hline $20-24$ & 749 & $333(44.5)$ & $118(15.8)$ & $245(32.7)$ & $73(9.7)$ & $96(12.8)$ \\
\hline $25-29$ & 621 & $192(30.9)$ & $54(8.7)$ & $130(20.9)$ & $37(6.0)$ & $49(7.9)$ \\
\hline $30-39$ & 945 & $220(23.3)$ & $59(6.2)$ & $133(14.1)$ & $41(4.3)$ & $45(4.8)$ \\
\hline$\geq 40$ & 1041 & $169(16.2)$ & $41(3.9)$ & $84(8.1)$ & $41(3.9)$ & $20(1.9)$ \\
\hline Missing & 166 & $49(29.5)$ & $12(7.2)$ & $31(18.7)$ & $9(5.4)$ & $14(8.4)$ \\
\hline Total & 4043 & 1207 (29.9) & $385(9.5)$ & 806 (19.9) & $259(6.4)$ & $305(7.5)$ \\
\hline
\end{tabular}

${ }^{1}$ High risk: HPV 16, 18, 31, 33, 35, 39, 45, 51, 52, 56, 58, 59.

${ }^{2}$ Probably/possibly carcinogenic: HPV 26, 53, 66, 67, 68, 70, 73, 82, 30, 69, 85. 
Pap test results were available for 4,031 women of whom $92.7 \%$ had normal cytology, $5.7 \%$ were diagnosed with a low-grade cervical lesion (ASCUS and LSIL) and $1.4 \%$ with a high-grade cervical lesion (ASC-H, HSIL, AGC, or SCC). The highest rate of abnormal cytology (2.3\%) was found in women aged 25 to 29 and this rate was below $2 \%$ in all other age groups (data not shown).

There were very few non-Aboriginal women less than 20 years of age that presented for cervical screening and consequently, statistical comparisons of ethnic groups did not include females less than 20 years of age. Among women 20 years of age and older, there were no statistically significant differences between Inuit and nonAboriginal (reference group) women regarding the prevalence of any HPV (OR: 1.19, 95\% Cl: 0.92-1.54), highrisk HPV (OR: 1.06, 95\% Cl: 0.78-1.44), HPV 16 and 18 (OR: 0.81, 95\% Cl: 0.51-1.27), or multiple HPV infections (OR: $1.23,95 \% \mathrm{Cl}: 0.73-2.06$ ) (Table 2). HPV 31 is the only type that was significantly more frequent among Inuit than non-Aboriginal women (OR: $3.95,95 \% \mathrm{Cl}: 1.24-12.54$, data not shown). There was no difference in the overall occurrence of cervical abnormalities $(p$-value $=0.17)$ between non-Aboriginal and Inuit women (data not shown).

Table 2: Comparison of human papillomavirus (HPV) prevalence by ethnicity among Inuit and nonAboriginal women presenting for cervical cancer screening in Nunavut, January 2008 to March 2009 $(\mathrm{N}=4,016)$

\begin{tabular}{|c|c|c|c|}
\hline \multirow{2}{*}{ HPV Result } & \multicolumn{2}{|c|}{ Ethnicity $^{1}$} & \multirow{2}{*}{ OR (95\% CI) } \\
\cline { 2 - 3 } & $\begin{array}{c}\text { Non- } \\
\text { Aboriginal }^{3} \\
(\mathrm{~N}=420)\end{array}$ & $\begin{array}{c}\text { Inuit } \\
(\mathrm{N}=3,596)\end{array}$ & \\
\hline Any & $89(21.2 \%)$ & $1114(31.0 \%)$ & $1.19(0.92-1.54)$ \\
\hline Multiple & $22(5.2 \%)$ & $362(10.1 \%)$ & $1.23(0.73-2.06)$ \\
\hline High-risk & $57(13.6 \%)$ & $747(20.8 \%)$ & $1.06(0.78-1.44)$ \\
\hline HPV 16/18 & $24(5.7 \%)$ & $280(7.8 \%)$ & $0.81(0.51-1.27)$ \\
\hline
\end{tabular}

${ }^{1}$ First Nations and Missing ethnicity excluded from analysis.

${ }^{2} \mathrm{OR}$ : odds ratio; CI: confidence interval

${ }^{3}$ Reference group

${ }^{4}$ High-risk: HPV 16, 18, 31, 33, 35, 39, 45, 51, 52, 56, 58, 59.

There were 1,207 women who were positive for any type of HPV, with a total of 1,761 infections (accounting for women positive for multiple types). Infections with species a09 were most frequent (36.9\% of infections), including HPV 16 (14.7\%), HPV 31 (7.1\%) and HPV 58 (4.7\%). Species a07 was the second most common species $(21.0 \%$ of infections), of which HPV 59 (5.2\%) was the most prevalent type, followed by HPV 45 (4.4\%), HPV 39 (4.0\%) and HPV 18 (3.2\%) (Table 3). 
Table 3: Distribution of human papillomavirus (HPV) infections by type and species among specimens positive for any type of HPV in women presenting for cervical cancer screening in Nunavut, January 2008 to March $2009(\mathrm{~N}=1,761)^{1}$

\begin{tabular}{|l|c|c|}
\hline \multirow{4}{*}{ Species } & $\begin{array}{c}\text { HPV } \\
\text { Type }\end{array}$ & N (\%) \\
\hline \multirow{4}{*}{ a01 } & 42 & $52(3.0)$ \\
\cline { 2 - 3 } & 32 & $8(0.5)$ \\
\cline { 2 - 3 } & Total a01 & $60(3.4)$ \\
\hline \multirow{4}{*}{ a03 } & 81 & $64(3.6)$ \\
\cline { 2 - 3 } & 72 & $46(2.6)$ \\
\cline { 2 - 3 } & 89 & $36(2.0)$ \\
\cline { 2 - 3 } & 62 & $34(1.9)$ \\
\cline { 2 - 3 } & Total a03 & $\begin{array}{c}258 \\
(14.7)\end{array}$ \\
\hline \multirow{5}{*}{ a05 } & 51 & $69(3.9)$ \\
\cline { 2 - 3 } & 69 & $8(0.5)$ \\
\cline { 2 - 3 } & 26 & $1(0.1)$ \\
\cline { 2 - 3 } & 82 & $1(0.1)$ \\
\cline { 2 - 3 } & Total a05 & $79(4.5)$ \\
\hline
\end{tabular}

\begin{tabular}{|c|c|c|}
\hline \multirow{4}{*}{ Species } & $\begin{array}{c}\text { HPV } \\
\text { Type }\end{array}$ & N (\%) \\
\hline \multirow{5}{*}{ a06 } & 66 & $75(4.3)$ \\
\cline { 2 - 3 } & 56 & $47(2.7)$ \\
\cline { 2 - 3 } & 53 & $25(1.4)$ \\
\cline { 2 - 3 } & 30 & $8(0.5)$ \\
\cline { 2 - 3 } & Total a06 & $\begin{array}{c}155 \\
(8.8)\end{array}$ \\
\hline \multirow{5}{*}{ a07 } & 59 & $92(5.2)$ \\
\cline { 2 - 3 } & 45 & $77(4.4)$ \\
\cline { 2 - 3 } & 39 & $71(4.0)$ \\
\cline { 2 - 3 } & 18 & $57(3.2)$ \\
\cline { 2 - 3 } & 70 & $49(2.8)$ \\
\cline { 2 - 3 } & 68 & $17(1.0)$ \\
\cline { 2 - 3 } & 85 & $7(0.4)$ \\
\cline { 2 - 3 } & Total a07 & $\begin{array}{c}370 \\
(21.0)\end{array}$ \\
\hline \multirow{5}{*}{ a08 } & 40 & $20(1.1)$ \\
\hline & 43 & $7(0.4)$ \\
\hline & 91 & $1(0.1)$ \\
\hline & Total a08 & $28(1.6)$ \\
\hline
\end{tabular}

\begin{tabular}{|c|c|c|}
\hline Species & $\begin{array}{l}\text { HPV } \\
\text { Type }\end{array}$ & N (\%) \\
\hline \multirow{8}{*}{ a09 } & 16 & $259(14.7)$ \\
\hline & 31 & $125(7.1)$ \\
\hline & 58 & $82(4.7)$ \\
\hline & 67 & $58(3.3)$ \\
\hline & 33 & $49(2.8)$ \\
\hline & 52 & $46(2.6)$ \\
\hline & 35 & $31(1.8)$ \\
\hline & Total a09 & $650(36.9)$ \\
\hline \multirow{6}{*}{ a10 } & 6 & $53(3.0)$ \\
\hline & 11 & $13(0.7)$ \\
\hline & 44 & $13(0.7)$ \\
\hline & 74 & $13(0.7)$ \\
\hline & 13 & $7(0.4)$ \\
\hline & Total a11 & 99 (5.6) \\
\hline a11 & 73 & $20(1.1)$ \\
\hline a13 & 54 & $27(1.5)$ \\
\hline a14 & 90 & $15(0.9)$ \\
\hline
\end{tabular}

${ }^{1}$ The 1,207 HPV positive women had a total of 1,761 HPV infections (i.e. each HPV type was counted individually).

\section{Association between HPV type and cervical cytological results}

HPV 16 was the most common type, being present in $50.9 \%$ of specimens with a high-grade cervical lesion (Table 4, all ethnic groups combined). HPV 18 was found in 5.5\% of high-grade lesions. HPV 16 was associated with the highest risk of being diagnosed with a high-grade lesion ( $\mathrm{OR}=16.8,95 \% \mathrm{Cl}$ : 9.8-29.0), followed by HPV 35 (OR=11.4, 95\% Cl: 3.9-34.0), HPV 45 (OR=6.7, 95\% Cl: 2.8-16.2) and HPV 18 (OR=4.2, 95\% Cl: 1.3-13.8); however, confidence intervals for these types overlapped so the differences between them are not statistically significant. Women who were positive for HPV 31, 33, 39, 51, 52, 56 and 59 were not at statistically higher risk of high-grade lesions (Table 4). 
Table 4: Association of high-grade cervical lesions with high-risk human papillomavirus (HPV) infections, all ethnic groups combined

\begin{tabular}{|c|c|c|c|c|}
\hline \multirow{2}{*}{\begin{tabular}{c}
\multirow{2}{*}{$\begin{array}{l}\text { HPV } \\
\text { type }\end{array}$} \\
\cline { 2 - 4 }
\end{tabular}} & $\begin{array}{l}|c| \\
\text { Normal }^{2} \\
(\mathrm{n}=3,747)\end{array}$ & $\begin{array}{l}\text { Low-grade } \\
(\mathrm{n}=229)\end{array}$ & $\begin{array}{l}\text { High-grade } \\
(\mathrm{n}=55)\end{array}$ & \multirow{2}{*}{ OR (95\% Cl) } \\
\cline { 2 - 4 } & $\mathrm{N}(\%)$ & $\mathrm{N}(\%)$ & $\mathrm{N}(\%)$ & \\
\hline $16 / 18$ & $216(5.8)$ & $60(26.2)$ & $29(52.7)$ & $15.0(8.7-25.7)$ \\
\hline 16 & $178(4.8)$ & $53(23.1)$ & $28(50.9)$ & $16.8(9.8-29.0)$ \\
\hline 18 & $42(1.1)$ & $12(5.2)$ & $3(5.5)$ & $4.2(1.3-13.8)$ \\
\hline 31 & $101(2.7)$ & $21(9.2)$ & $3(5.5)$ & $1.8(0.6-5.9)$ \\
\hline 33 & $32(0.9)$ & $16(7.0)$ & $1(1.8)$ & $1.5(0.2-11.2)$ \\
\hline 35 & $22(0.6)$ & $5(2.2)$ & $4(7.3)$ & $11.4(3.9-34.0)$ \\
\hline 39 & $54(1.4)$ & $16(7.0)$ & $1(1.8)$ & $1.03(0.1-7.6)$ \\
\hline 45 & $58(1.5)$ & $13(5.7)$ & $6(10.9)$ & $6.7(2.8-16.2)$ \\
\hline 51 & $50(1.3)$ & $19(8.3)$ & $0(0.0)$ & - \\
\hline 52 & $38(1.0)$ & $7(3.1)$ & $1(1.8)$ & $1.6(0.2-12.0)$ \\
\hline 56 & $36(1.0)$ & $9(3.9)$ & $2(3.6)$ & $3.3(0.8-13.9)$ \\
\hline 58 & $61(1.6)$ & $17(7.4)$ & $4(7.3)$ & $3.9(1.4-11.1)$ \\
\hline 59 & $71(1.9)$ & $18(7.9)$ & $3(5.5)$ & $2.5(0.8-8.2)$ \\
\hline
\end{tabular}

${ }^{\mathrm{O}} \mathrm{OR}$ : odds ratio; CI: confidence interval

${ }^{2}$ Reference category

${ }^{3}$ Low-grade lesions: atypical squamous cells of undetermined significance and low-grade squamous intraepithelial lesion.

${ }^{4} \mathrm{High}$-grade lesions: atypical squamous cells - cannot exclude high-grade squamous epithelial lesion; high-grade squamous epithelial lesion; squamous cell carcinoma; atypical glandular cells, not otherwise specified.

\section{Discussion}

This analysis identified that HPV infection is more prevalent among women living in Nunavut than in many other geographical regions of Canada where HPV prevalence has been studied $(9,10,12,13,15) ; 29.9 \%$ of all women tested during the surveillance period were positive for one or more HPV types. Of those positive, HPV 16 was the most common type (14.7\% of infections) followed by HPV 31 (7.1\% of infections). The prevalence of HPV infections was greatest among women less than 25 years of age of any ethnicity. The prevalence of HPV was $31.0 \%$ among Inuit women compared to $21.2 \%$ among non-Aboriginal women, but this difference was not statistically significant.

Other HPV prevalence studies conducted among women in regions of Canada with large Inuit populations have found similar results. In their cross-sectional study of HPV prevalence among Inuit and non-Inuit women aged 13 to 79 in Nunavut, Healey et al (2001) found a 25.8\% prevalence of oncogenic HPV, with those aged 13 to 20 having the highest odds of infection and no statistically significant difference between Inuit and non-Inuit women (8). Hamelin-Douglas et al (2008) conducted a study among Inuit women living in Nunavik (northern Quebec), reporting a prevalence of any HPV infection of $28.9 \%$ (high-risk HPV: $20.4 \%$ ); prevalence of any type of HPV was highest (58\%) in those younger than 20 years of age (high-risk HPV: 46.9\%) (13).

HPV 16 was associated with the highest odds of high-grade cervical lesions (ASC-H, AGC, HSIL and SCC). Although the prevalence of HPV 18 was low (1.4\%), this type was associated with a significant risk of high-grade lesions. (Note: These results must be interpreted with caution due to the small number of infections detected with this type.) Approximately $52 \%$ of the specimens with high-grade lesions were positive for HPV 16 and/or 18, a finding that is consistent with other North American studies as reported in a meta-analysis by Bosch et al (2008), 
who reported that the fraction of vaccine-preventable cervical cancer due to these types could be as high as $70 \%$ (21). The HPV vaccines currently in use in Canada protect against both types 16 and 18 and as such, the 2010 implementation of routine immunization for HPV in grade 6 girls in Nunavut should, in time, yield good results in reducing infection with these types and related abnormal cervical cytology. With evidence for cross-protection against related HPV types, the burden of infection with other species a07 and a09 types may be reduced as well $(19,22,23)$.

Without a longitudinal component, it is not possible to determine the persistence of type-specific infections and their impact on the risk of future abnormal cervical cytology. Over half $(51.4 \%)$ of the participants were 30 years of age or younger, as were the majority of women infected with high-risk or probably/possibly carcinogenic HPV. HPV infections in younger women are likely to be transient and thus may not result in a cancerous outcome (24). Future work in this population could examine persistence of type-specific infection and associated risk of highgrade cervical lesions.

No survey was administered during the surveillance period and therefore it is not possible to interpret the findings with respect to related risk factors such as sexual activity (e.g., number of partners, age of sexual debut and condom use), parity and smoking practices. These risk factors would be important to assess as cofactors in the acquisition and persistence of HPV infection in any future study conducted in this population.

There are several limitations in the surveillance methods used in this population. For example, this study represents only women who presented for Pap testing (primary screening or follow-up testing) in Nunavut between January 2008 and March 2009. Those who did not seek cervical screening may present different demographic and/or behavioural characteristics. No risk survey was used to examine cofactors for the acquisition of HPV or the development of cervical outcomes which limits the inferences that can be made. There were low numbers of observations in a number of categories used for comparison which restricts the explanatory power of the data and the relatively small number of non-Aboriginal women precludes adequate explanatory power for many sub-group analyses by ethnicity. It was not possible to perform detailed statistical analyses on First Nations women because of the small population living in Nunavut.

\section{Conclusion}

A large proportion of women who attend cervical screening in Nunavut tested positive for one or more types of HPV. Similar to other studies, HPV 16 was the most prevalent and showed the strongest association with highgrade cervical lesions. HPV 31 was the second most prevalent type, but its association with high-grade cervical dysplasia was not demonstrated.

HPV infection can be prevented with routine immunization and the associated high-grade cervical abnormalities in females can be mitigated through early detection with regular cervical screening. The results of this study provide a baseline of HPV prevalence that allows for the evaluation of the population-based HPV immunization program implemented in Nunavut in 2010, although it may take a decade or more of routine immunization with good coverage of the eligible population (grade 6 girls) before a significant impact on cervical screening results can be detected. Evaluation of public health programs such as HPV immunization can lead to evidence-informed interventions and improved health outcomes for the women of Nunavut.

\section{Acknowledgements}

The authors gratefully acknowledge the contribution of the Nunavut Department of Health and Social Services, Nunavut health care professionals and the women of Nunavut to this important surveillance initiative. 


\section{Conflict of interest}

None

\section{Funding}

None

\section{References}

(1) International Agency for Research in Cancer. IARC monographs on the evaluation of carcinogenic risks to humans: human papillomaviruses. 2007:670.

(2) Walboomers JMM, Jacobs MV, Manos MM, Bosch FX, Kummer JA, Shah KV, et al. Human papillomavirus is a necessary cause of invasive cervical cancer worldwide. J Pathol. 1999;189;12-19.

(3) Public Health Agency of Canada. Ontario/Nunavut Agency Regional Office Annual Report 2006-07. Ontario/Nunavut Agency Regional Office, Public Health Agency of Canada; 2007.

http://publications.gc.ca/collections/collection_2012/aspc-phac/HP2-8-2007-eng.pdf.

(4) Jayaraman G, Totten S, Perin M, Fang L, Remes O. Report on sexually transmitted infections in Canada: 2008.

Ottawa: Centre for Communicable Diseases and Infection Control, Infectious Disease Prevention and Control Branch, Public Health Agency of Canada; 2010. http://publications.gc.ca/collections/collection_2011/aspc-phac/HP37-10-2008eng.pdf.

(5) Healey S, Plaza D, Osborne G. A ten-year profile of cancer in Nunavut. Iqaluit: Government of Nunavut; 2003. http://pubs.aina.ucalgary.ca/health/55692E.pdf.

(6) Nunavut Department of Health and Social Services. Developing healthy communities: A public health strategy for Nunavut 2008-2013. Iqaluit: Government of Nunavut; 2008.

http://www.gov.nu.ca/sites/default/files/files/Public\%20Health\%20Strategy\%20-\%20English\%20final.pdf.

(7) Healey SM, Aronson K, Mao Y, Franco EL. Human papillomavirus and cervical dysplasia in Nunavut: Prelude to a screening strategy. Int J Circumpolar Health. 2004;63:(2):199-201.

(8) Healey SM, Aronson KJ, Mao Y, Schlecht NF, Mery LS, Ferenczy A, et al. Oncogenic human papillomavirus infection and cervical lesions in aboriginal women of Nunavut, Canada. Sex Transm Dis. 2001;28:694-700.

(9) Ratnam S, Franco EL, Ferenczy A. Human papillomavirus testing for primary screening of cervical cancer precursors. Cancer Epidemiol Biomarkers Prev. 2000;9:945-951.

(10) Moore RA, Ogilvie G, Fornika D, Moravan V, Brisson M, Amirabbasi-Beik M, et al. Prevalence and type distribution of human papillomavirus in 5,000 British Columbia women--implications for vaccination. Cancer Causes Control. 2009;20:1387-1396.

(11) Richardson H, Franco E, Pintos J, Bergeron J, Arella M, Tellier P. Determinants of low-risk and high-risk cervical human papillomavirus infections in Montreal University students. Sex Transm Dis. 2000;27:79-86.

(12) Sellors JW, Mahony JB, Kaczorowski J, Lytwyn A, Bangura H, Chong S, et al. Prevalence and predictors of human papillomavirus infection in women in Ontario, Canada. CMAJ. 2000;163:503-508.

(13) Hamlin-Douglas LK, Coutlée F, Roger M, Franco EL, Brassard P. Prevalence and age distribution of human papillomavirus infection in a population of Inuit women in Nunavik, Quebec. Cancer Epidemiol Biomarkers Prev. 2008;17:3141-3149.

(14) Young TK, McNicol P, Beauvais J. Factors associated with human papillomavirus infection detected by polymerase chain reaction among urban Canadian Aboriginal and non-Aboriginal women. Sex Transm Dis. 1997;24:293-298.

(15) Jiang Y, Brassard P, Severini A, Goleski V, Santos M, Leamon A, et al. Type-specific prevalence of Human Papillomavirus infection among women in the Northwest Territories, Canada. J infect Public Health. 2011;4:219-227.

(16) Zubach V, Smart G, Ratnam S, Severini A. Novel microsphere-based method for detection and typing of 46 mucosal human papillomavirus types. J Clin Microbiol. 2012;50:460-464.

(17) Bouvard V, Baan R, Straif K, Grosse Y, Secretan B, El Ghissassi F, et al. A review of human carcinogens--Part B: biological agents. Lancet Oncol. 2009;10:321-322.

(18) De Villiers E, Fauquet C, Broker TR, Bernard H, Zur Hausen H. Classification of papillomaviruses. Virology. 2004;324:17-27.

(19) Bonanni P, Boccalini S, Bechini A. Efficacy, duration of immunity and cross-protection after HPV vaccination: A review of the evidence. Vaccine. 2009;27:A46-A53.

(20) Solomon D, Davey D, Kurman R, Moriarty A, O'Connor D, Prey M, et al. The 2001 Bethesda System: Terminology for reporting results of cervical cytology. J Am Med Assoc. 2002;287:2114-2119.

(21) Bosch FX, Burchell AN, Schiffman M, Giuliano AR, de Sanjose S, Bruni L, et al. Epidemiology and natural history of Human Papillomavirus infections and type-specific implications in cervical neoplasia. Vaccine. 2008;26:K1-K16.

(22) Brown DR, Kjaer SK, Sigurdsson K, Iversen O, Mauricio H, Wheeler CM, et al. The impact of quadrivalent human papillomavirus (HPV; Types 6, 11, 16, and 18) L1 virus-like particle vaccine on infection and disease due to oncogenic nonvaccine HPV types in generally HPV-naive women aged 16-26 years. J Infect Dis. 2009;199:926-935. 
(23) Paavonen J, Naud P, Salmerón J, Wheeler CM, Chow S, Apter D, et al. Efficacy of human papillomavirus (HPV)-16/18 AS04-adjuvanted vaccine against cervical infection and precancer caused by oncogenic HPV types (PATRICIA): Final analysis of a double-blind, randomised study in young women. Lancet. 2009;374:301-314.

(24) Richardson H, Kelsall G, Tellier P, Voyer H, Abrahamowicz M, Ferenczy A, et al. The natural history of type-specific human papillomavirus infections in female university students. Cancer Epidemiol Biomarkers Prev. 2003;12:485-490. 